\title{
ERRATUM TO: ON THE DJL CONJECTURE FOR ORDER 6
}

\author{
Peter J. C. Dickinson
}

Abstract. In this note an erratum is provided to the article "On the DJL conjecture for order 6" by Naomi Shaked-Monderer, published in Operators and Matrices 11(1), 2017, 71-88. We will demonstrate and correct two errors in this article. The first error is in the statement of a proposition, which omits a certain category of extreme matrices. The second error is in the proof of a lemma. Fortunately the lemma itself is correct, and in this note we will in fact show that a stronger result holds.

Mathematics subject classification (2010): 15B48, 15A23.

Keywords and phrases: Copositive matrix, extremal matrix, minimal zeros, erratum.

\section{REFERENCES}

[1] N. ShaKed-Monderer, On the DJL conjecture for order 6, Operators and Matrices 11 (1), 71-88, 2017.

[2] M. Hall JR. And and M. Newman, Copositive and Completely Positive Quadratic Forms, Mathematical Proceedings of the Cambridge Philosophical Society, 59: 329-339, 1963.

[3] P. J. C. Dickinson, The Copositive Cone, the Completely Positive Cone and their Generalisations $\mathrm{PhD}$ Thesis, University of Groningen, 2013.

[4] R. Hildebrand, Minimal zeros of copositive matrices, Linear Algebra and Its Applications 459, 154-174, 2014. 